\title{
On The Bandwidth Dependency of Near-Field Effects in UWB Implant Body Area Networks \\ Muzaffer Kanaan ${ }^{1} \quad$ Memduh Suveren ${ }^{1} \quad$ Ömer Galip Saraçoğlu ${ }^{2}$
}

\author{
${ }^{1}$ Erciyes University, Dept. of Mechatronics Engineering, \\ Melikgazi 38039 KAYSERI - TURKEY \\ \{mkanaan, msuveren\}@erciyes.edu.tr
${ }^{2}$ Erciyes University, Dept. of Electrical and Electronic Engineering, Melikgazi 38039 KAYSERI - TURKEY saracog@erciyes.edu.tr

\begin{abstract}
We describe the results of our investigations into the bandwidth dependency of near-field effects in UWB implant body area networks. It has previously been proposed in the literature that the near-field region of an implant UWB antenna expands as a result of the interaction of the antenna with body tissues. However, the bandwidth dependency of this near-field effect has not been fully investigated. Our results shed light into this matter, which should be helpful in optimizing the quality of an implant UWB link.
\end{abstract}

\section{Categories and Subject Descriptors}

C.2.1 [Computer-Communication Networks]: Network Architecture and Design - Wireless communication.

\section{General Terms}

Design

\section{Keywords}

Body Area Networks, Ultra Wide Band, Wireless Communication

\section{INTRODUCTION}

Body area networks (BANs) are a new and growing area of wireless communications, specifically focusing on low-power networking between wearable and implantable sensor nodes of small form factor. Potential application areas range from health and wellness to personalized entertainment and military applications [1]. The general system scenario is as shown in Figure 1 below. A number of sensor nodes are shown in the figure, which can be deployed on the body surface, implanted or even off the body. However, regardless of where the sensors are placed, it is generally assumed that they all communicate to one central node (which can be either on or off the body), known as a "base station" or "hub". The base station in a BAN is simply a node that has the capability to communicate to a wide area network. A typical example of a base station would be a smart phone with $3 \mathrm{G}$ or $4 \mathrm{G}$ cellular connectivity. The base station relays the data from the sensors on to an information sink (for example, a telemedicine server at the local hospital for a remote health

Permission to make digital or hard copies of all or part of this work for personal or classroom use is granted without fee provided that copies are not made or distributed for profit or commercial advantage and that copies bear this notice and the full citation on the first page. To copy otherwise, to republish, to post on servers or to redistribute to lists, requires prior specific permission and/or a fee.

UWBAN-2013 2013, September 30-October 02

Copyright $\odot 2013$ ICST 978-1-936968-89-3

DOI 10.4108/icst.bodynets.2013.253926 monitoring application).

Ultra wide band (UWB) technology is being considered for a number of different applications in BANs, ranging from accurate indoor localization for mentally ill and disabled patients [2] to better-quality capsule endoscopy [3]. It is also conceivable that UWB-based medical implant communications will also be important for next-generation medical imaging and robotic surgery applications [4]. In order to optimize the UWB transceiver

designs for these types of applications, better understanding of UWB propagation in implant environments is needed. Our goal in this paper is to contribute to this understanding.

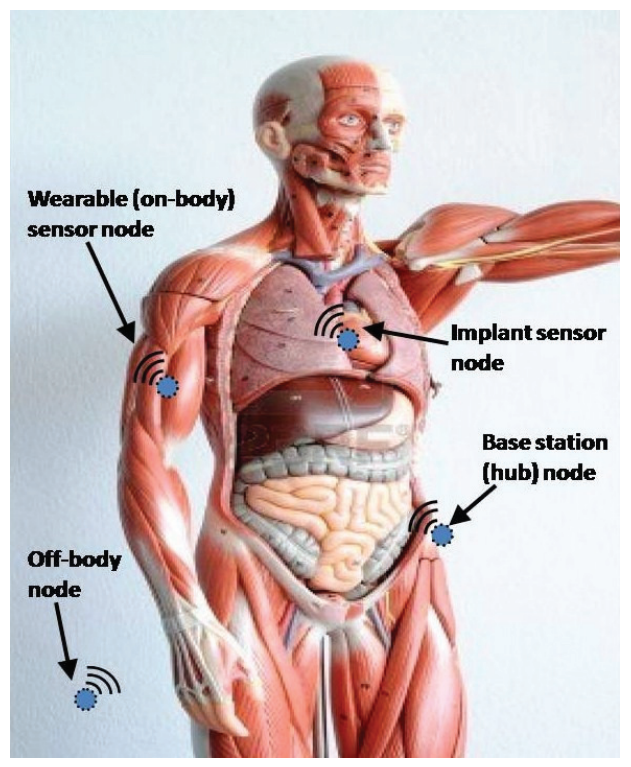

Figure 1 Conceptual view of a body area network (depicted here on a 3D anatomical model)

There have been several previous studies on UWB propagation modeling for biomedical implant scenarios. In [5], the authors modeled the UWB propagation in implant scenarios; these studies indicated that UWB communication with an implant is feasible for low-data rate applications. In [6], the researchers numerically modeled the propagation between an implant embedded in the human chest and a sensor on the surface of the body; this study also included the multipath parameters that were extracted from 
the simulation results. In [7], the authors studied the UWB propagation in implant scenarios and postulated the existence of a near-field region at distances close to the antenna. Related works having to do with UWB antenna design and performance evaluation for implant applications can be found in [8] and [9]

The starting point for our work was [7], and specifically the path loss models reported therein. In this work, the authors reported increased path-loss exponents at distances close to the body surface. These results were observed at all implant depths. From these results, the authors postulated the existence of a nonradiating near-field region close to the body surface and proposed that receiving antennas be positioned as close to the body surface as possible in order to exploit the near-field and improve link quality. However, for practical applications such as wireless medical telemetry, having a wide non-radiating near-field region is undesirable, as it essentially represents a waste of transmitter power. Positioning the antenna so close to the body surface is also undesirable from a usability standpoint due to possible skin irritations resulting from attachment of the antenna on the skin, or just plain user annoyance.

All this leads to an interesting question: is it possible to reduce the near-field effects in UWB implant communications by adjusting system parameters, such as the bandwidth of the UWB signal? If the near-field effects could be reduced by intelligently adjusting system parameters, more of the transmitter power is radiated; this enhances the power efficiency of the implant wireless sensor. In addition, it becomes easier to integrate BAN platforms with other consumer electronic devices which are not typically deployed close to the body surface (such as tablets and smart phones), paving the way for a more attractive user experience. While it may be argued that the problem of the near-field region has more to do with the antenna design itself, the antenna, once designed and embedded in human tissue, is typically a static structure whose parameters cannot be changed easily. Therefore, it is logical to investigate whether other system parameters, such as signal bandwidth (which are easier to change), can be adjusted to reduce near-field effects. In this paper, we have attempted to address this issue through an analysis of 3-D electromagnetic simulation results.

The rest of this paper is divided into three sections. In section 2, we describe the simulation scenarios that we used. Section 3 presents our results and analysis. Section 4 concludes the paper.

\section{SIMULATION SCENARIO}

Finite Difference Time Domain (FDTD) electromagnetic simulations were carried out on a 3-D voxel model of the human body developed under the Visible Human Project [10]. FDTD techniques are especially appropriate for UWB studies, since the response of a system to a broadband pulse can be easily captured in one simulation. The tool used to perform the simulations was the XFDTD ${ }^{\mathrm{TM}}$ software from Remcom, Inc.

The resolution of the body model used was $2 \mathrm{~mm}$. The human body mesh data was adjusted until just the section of interest (i.e. human chest and torso sections) remained. Figure 2 shows the body model used. An elliptic disc dipole antenna was placed in the chest close to the heart, similar to [7], as shown in Figure 3. The FDTD cell size used was also $2 \mathrm{~mm}$. Considering the tissue types in the region of interest and the frequency range being used, this cell size satisfied the Courant limit. The antenna was excited with a Gaussian pulse with a $-6 \mathrm{~dB}$ bandwidth of 500 , 750 and $1000 \mathrm{MHz}$ respectively. Since our objective was to study the effects of signal bandwidth on the near-field effects, the input signal was not modulated with a carrier frequency. The Perfectly Matched Layer (PML) absorbing boundary condition with seven layers was used to limit the simulation region of interest. The frequency-dependent material properties were modeled using a four-pole Cole-Cole model as given by

$$
\begin{aligned}
\hat{\varepsilon}(\omega) & =\varepsilon^{\prime}(\omega)+j \varepsilon^{\prime \prime}(\omega) \\
& =\varepsilon_{\infty}+\sum_{k=1}^{4} \frac{\Delta \varepsilon_{k}}{1+\left(j \omega \tau_{k}\right)^{\left(1-\alpha_{k}\right)}}+\frac{\sigma_{i o n}}{j \omega \varepsilon_{0}}
\end{aligned}
$$

where $\varepsilon^{\prime}(\omega)$ and $\varepsilon^{\prime \prime}(\omega)$ represent the frequency-dependent dielectric constant and dielectric loss respectively. The parameter $\varepsilon_{\infty}$ represents the dielectric constant at infinite frequency, $\Delta \varepsilon_{k}$ is the magnitude of the dispersion, $\tau_{k}$ is the relaxation time and $\alpha_{k}$ is the parameter that models the broadening of the dispersion. Finally, $\sigma_{i o n}$ is the static ionic conductivity and $\varepsilon_{0}$ represents the permittivity of free space. The parameter values for the different tissue types were taken from the IT'IS tissue database [11].

As part of the simulations, electric and magnetic field intensities were calculated at points on a straight line radially away from the center point of the antenna. The field values were measured using software-defined field probes. The antenna was implanted at depths of 35 and $45 \mathrm{~mm}$. The Poynting vector $\left(\right.$ in $\mathrm{W} / \mathrm{m}^{2}$ ) for was calculated at the points of interest, as defined by

$$
\mathbf{P}_{x, y, z}(t)=\mathbf{E}_{x, y, z}(t) \times \mathbf{H}_{x, y, z}(t)
$$

where $\mathbf{E}_{x, y, z}(t)$ and $\mathbf{H}_{x, y, z}(t)$ represent the time-dependent electric and magnetic field intensities respectively. The Poynting vector is then integrated to obtain the UWB signal energy density (in $\mathrm{J} / \mathrm{m}^{2}$ ) as given by

$$
e=\int_{t}\left|\mathbf{P}_{x, y, z}(t)\right| d t
$$

For all the bandwidth values, a total of 70 field probes were defined. The probes were at various distances from the body surface. The distances, $r$, were defined as a ratio of the depth of the implant from the body surface, $r_{0}$, where $r_{0}=35 \mathrm{~mm}$ or $45 \mathrm{~mm}$ (for example, $r / r_{0}=1$ represents the body surface regardless of the implant depth). The values of the energy density were all normalized to $1 \mathrm{~J}$ and are expressed as $\mathrm{dBJ} / \mathrm{m}^{2}$ in the results that follow. 


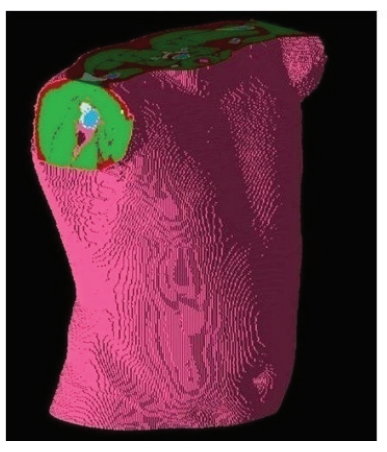

(a)

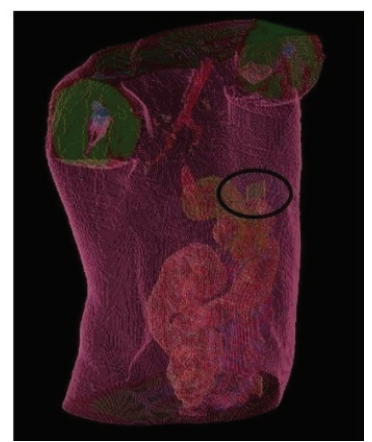

(b)

Figure 2 3-D body model used for the simulations: (a) General view, (b) A more transparent view showing the antenna implanted in the chest (indicated in black oval).

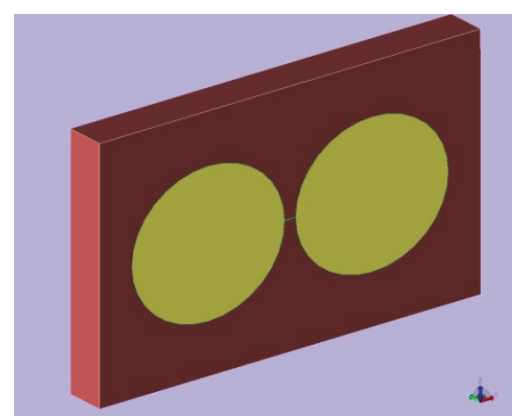

Figure 3 Elliptic dipole antenna [7]

\section{RESULTS AND DISCUSSION}

The first results of our simulations are as shown in Figure 4. These results are for an implant depth of $35 \mathrm{~mm}$ below the body surface. It can be clearly seen from these results that increasing the bandwidth results in higher energy density values near the body surface (such as the interval $1 \leq r / r_{0}<2.2$ ). In order to derive a path loss model from these results, the following wellknown template for distance-dependent path loss is used [12]

$$
P(r)=10 n \log \left(\frac{r}{r_{0}}\right)+\alpha
$$

where $n$ is the path loss exponent and $\alpha$ is a power-scaling constant. These values were derived using least-squares regression based on the energy density values calculated. In principle, the regression could have been performed using a single parameter set $\left(n\right.$ and $\alpha$ ) for all values of $r / r_{0}$. However, we found that a piecewise fit for different intervals of $r / r_{0}$ gave more accurate results; this was also the strategy adopted in [7]. However, in contrast to [7], we found that allowing the $\alpha$ values to also change with the different intervals resulted in a more accurate fit (although it made the model slightly more complicated).

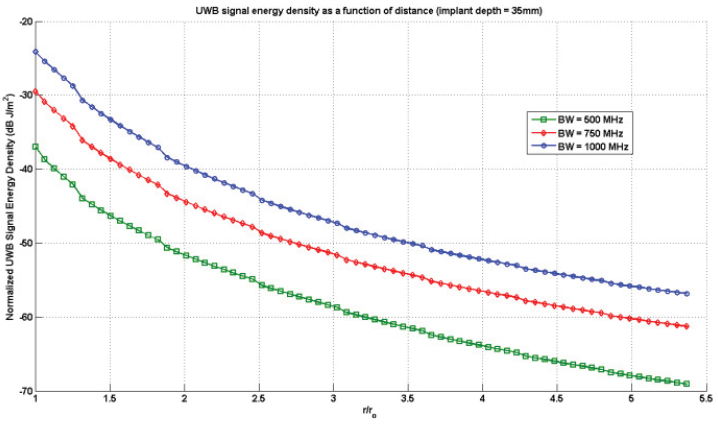

Figure 4 UWB signal energy density as a function of distance for different signal bandwidth values (implant depth: $\mathbf{3 5} \mathbf{~ m m}$ ).

Table 1 and Table 2 below give the model parameters for $35 \mathrm{~mm}$ implant depth.

Table 1 Values of the path loss exponent ( $n$ ) for different bandwidth values (implant depth: 35 mm)

\begin{tabular}{|c|c|c|c|}
\hline \multirow{2}{*}{ Interval } & \multicolumn{3}{|c|}{ Bandwidth values } \\
\cline { 2 - 4 } & $\mathbf{5 0 0} \mathbf{~ M H z}$ & $\mathbf{7 5 0} \mathbf{~ M H z}$ & $\mathbf{1 0 0 0} \mathbf{~ M H z}$ \\
\hline $1<r / r_{0}<2.2$ & 4.62 & 4.82 & 5.08 \\
\hline $2.2<r / r_{0}<3.2$ & 4.22 & 4.22 & 4.46 \\
\hline $3.3<r / r_{0}<4.3$ & 4.13 & 3.87 & 3.83 \\
\hline $4.3<r / r_{0}<5.4$ & 3.98 & 3.64 & 3.52 \\
\hline
\end{tabular}

Table 2 Values of the power scaling constant ( $\alpha$ ) for different bandwidth values (implant depth: 35 mm)

\begin{tabular}{|c|c|c|c|}
\hline \multirow{2}{*}{ Interval } & \multicolumn{3}{|c|}{ Bandwidth values } \\
\cline { 2 - 4 } & $\mathbf{5 0 0} \mathbf{~ M H z}$ & $\mathbf{7 5 0} \mathbf{~ M H z}$ & $\mathbf{1 0 0 0} \mathbf{~ M H z}$ \\
\hline $1<r / r_{0}<2.2$ & 37.72 & 29.77 & 24.13 \\
\hline $2.2<r / r_{0}<3.2$ & 38.60 & 31.48 & 26.06 \\
\hline $3.3<r / r_{0}<4.3$ & 39.04 & 33.22 & 29.19 \\
\hline $4.3<r / r_{0}<5.4$ & 40.07 & 34.73 & 31.21 \\
\hline
\end{tabular}

Figure 5 shows the variation of the signal energy density as a function for the implant depth of $45 \mathrm{~mm}$ for different bandwidth values. 


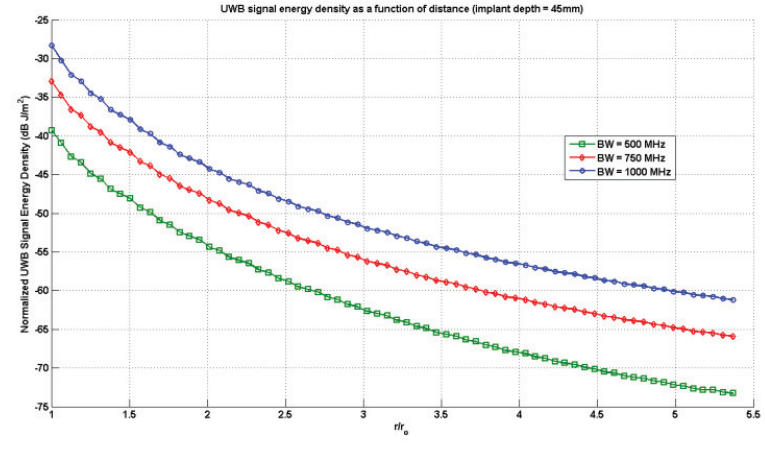

Figure 5 UWB signal energy density as a function of distance for different signal bandwidth values (implant depth: $45 \mathrm{~mm}$ ).

Table 3 and Table 4 give the model parameters for the $45-\mathrm{mm}$ implant depth at different bandwidth values.

Table 3 Values of the path loss exponent $(n)$ for different bandwidth values (implant depth: $45 \mathrm{~mm}$ )

\begin{tabular}{|c|c|c|c|}
\hline \multirow{2}{*}{ Interval } & \multicolumn{3}{|c|}{ Bandwidth values } \\
\cline { 2 - 4 } & $\mathbf{5 0 0} \mathbf{~ M H z}$ & $\mathbf{7 5 0} \mathbf{~ M H z}$ & $\mathbf{1 0 0 0} \mathbf{~ M H z}$ \\
\hline $1<r / r_{0}<2.2$ & 4.77 & 4.81 & 4.98 \\
\hline $2.2<r / r_{0}<3.2$ & 4.71 & 4.42 & 4.27 \\
\hline $3.3<r / r_{0}<4.3$ & 4.45 & 4.03 & 3.82 \\
\hline $4.3<r / r_{0}<5.4$ & 4.11 & 3.79 & 3.59 \\
\hline
\end{tabular}

Table 4 Values of the power scaling constant $(\alpha)$ for different bandwidth values (implant depth: $45 \mathrm{~mm}$ )

\begin{tabular}{|c|c|c|c|}
\hline \multirow{2}{*}{ Interval } & \multicolumn{3}{|c|}{ Bandwidth values } \\
\cline { 2 - 4 } & $\mathbf{5 0 0} \mathbf{~ M H z}$ & $\mathbf{7 5 0} \mathbf{~ M H z}$ & $\mathbf{1 0 0 0} \mathbf{~ M H z}$ \\
\hline $1<r / r_{0}<2.2$ & 39.85 & 33.70 & 29.19 \\
\hline $2.2<r / r_{0}<3.2$ & 39.92 & 34.86 & 31.35 \\
\hline $3.3<r / r_{0}<4.3$ & 41.26 & 36.81 & 33.59 \\
\hline $4.3<r / r_{0}<5.4$ & 43.38 & 38.28 & 35.00 \\
\hline
\end{tabular}

The results given above clearly indicate that the path loss exponent is higher at points close to the body surface. As one gets further away from the body surface, the path-loss exponent drops in value. The higher values of the path-loss exponent at points close to the body surface (for example, points within the interval $1<r / r_{0}<2.2$ ) indicate the existence of a near-field region close to the body surface; this is in line with the observations outlined in [7]. However, we also observe that the values of the path-loss exponent close to the body surface also increase as we increase the bandwidth. These results suggest that increasing the bandwidth of the input UWB signal results in a more pronounced near-field effect. Increasing the bandwidth obviously results in greater information-carrying capacity; however, as the results above illustrate, it may result in less favorable propagation characteristics.

An intuitive explanation for these observations can be given as follows. Electromagnetic waves travel at a constant speed known as the phase velocity in free space. If the medium is lossy, this velocity becomes frequency-dependent. The human body certainly qualifies as a lossy medium, owing to the conductivity characteristics of different body tissues. A lossy medium is also known as a dispersive medium. In a dispersive medium the lower frequency components of the signal will travel slower with respect to higher-frequency components [13]. Therefore, a UWB signal with a higher bandwidth will encounter more losses and distortion, as opposed to one with a narrower bandwidth.

\section{CONCLUSION}

In this paper, we presented the initial results of our efforts to quantify the relationship between signal bandwidth and near-field effects in UWB implant body area networks. Our results indicate that the near-field effects are reduced as the signal bandwidth is reduced. Using a signal with a wider bandwidth obviously increases the information-carrying capacity; however, it may also result in more pronounced near-field effects, and thus less favorable propagation conditions. Our overall conclusion is that there is a trade-off between the information-carrying capacity of the system and the propagation conditions desired. This trade-off needs to be taken into account by the system designer seeking to develop a UWB-based implant communications system.

\section{ACKNOWLEDGMENTS}

The authors would like to thank the anonymous reviewers for their time and thoughtful comments. This work was supported by the Erciyes University Research Fund under project FBA-123885 .

\section{REFERENCES}

[1] Li, H-B., Yazdandoost, K. Y., Zhen, B. 2010. Wireless Body Area Network. River Publishers, Aalborg, Denmark.

[2] Stelios, M. A., et al. 2008. An Indoor Localization Platform for Ambient Assisted Living Using UWB. In Proceedings of the 6th International Conference on Advances in Mobile Computing and Multimedia (Linz, Austria, November 24-26, 2008). MoMM 2008. ACM, New York, NY, 178-182. DOI: $10.1145 / 1497185.1497223$.

[3] Khaleghi, A., Chávez-Santiago, R., Balasingham, I. 2010. Ultra-wideband pulse-based data communications for medical implants. IET Communications, 4, 5 (February 2010) 1889-1897, DOI: 10.1049/iet-com.2009.0692.

[4] Kanaan, M. Ranging Based on Maximum Likelihood Techniques for Ultra Wide Band Medical Implants, In Proceedings of the IEEE $22^{\text {nd }}$ International Symposium on Personal Indoor Mobile Radio Communications (Toronto, Canada, Sept. 11-14, 2011) . PIMRC'11. IEEE, New York, NY, 2234-2238. DOI: 10.1109/PIMRC.2011.6139915.

[5] Ghildiyal, A. Godara, B., Amara, K., Dal Molin, R., Amara, A., "Ultra Wideband for in and on-body medical implants: A study of the limits and new opportunities,", In Proceedings of the 5th European Conference on Antennas and Propagation (Rome, Italy, April 11-15 2011). EuCAP 2011. IEEE, New York, NY 3778-3782.

[6] Wang Q., Masami, K., Wang, J., Channel modeling and BER performance for wearable and implant UWB body area links on chest, In Proceedings of the IEEE International 
Conference on Ultra-Wideband, 2009 (Vancouver, Canada, September 9-11, 2009). ICUWB 2009. IEEE, New York, NY 316-320. DOI: 10.1109/ICUWB.2009.5288734

[7] Khaleghi A., Balasingham I. 2009., Improving In-Body Ultra Wideband Communication Using Near-Field Coupling of the Implanted Antenna, Microwave and Optical Technology Letters, 51,3 (March 2009), 585-589, DOI: 10.1002/mop.24126.

[8] Dissanayake, T., Yuce, M.R., Chee Ho. 2009. Design and Evaluation of a Compact Antenna for Implant-to-Air UWB Communication, IEEE Antennas and Wireless Propagation Letters, 8 (January 2009), 153-156, DOI: 10.1109/LAWP.2009.2013370

[9] Eesuola, A.; Chen, Y.; Tian, G.Y., Novel ultra-wideband antennas for in-body wireless communication and medical imaging applications, In Proceedings of the 5th European
Conference on Antennas and Propagation (Rome, Italy, April 11-15 2011). EuCAP 2011. IEEE, New York, NY 3129-3132.

[10] Ackerman, M. J. 1991. Viewpoint: The Visible Human Project, Journal Biocommunication, 18,2, p. 14.

[11] IT'IS Tissue Database, http://www.itis.ethz.ch/itis-forhealth/tissue-properties/database/ (last accessed June 10, 2013).

[12] Oppermann, I., Hämäläinen, M., Iinatti, J., 2004. UWB Theory and Applications, John Wiley \& Sons, Chichester, England.

[13] Cheng, David K. 1992. Fundamentals of Engineering Electromagnetics, Prentice-Hall 